\title{
EDITORIAL
}

\section{Pandemic unpreparedness?}

\author{
Analysis of national influenza pandemic preparedness plans reveals that the preparations for \\ an effective response in the early stages of a pandemic, while a vaccine is still being \\ prepared, are giving cause for concern.
}

Since the mid-1990s, influenza viruses of the H5N1 subtype have become widespread in avian populations in southeast Asia. What is particularly significant about this virus is that it is unusual for highly pathogenic avian influenza to be so widespread and to have the ability to infect wild bird populations. The extended distribution of the $\mathrm{H} 5 \mathrm{~N} 1$ virus undoubtedly elevates the risk of a global human influenza pandemic and the WHO now considers that this risk is higher than at any time since the last pandemic in 1968. When the pandemic risk that this virus poses is combined with its mortality rate in humans (estimated at 58\%), there is little doubt that the impact of an $\mathrm{H} 5 \mathrm{~N} 1$ pandemic could be catastrophic.

When the next pandemic does strike, vaccination will have a key role in its control at both the national and international levels. However, vaccine use will be limited in the early stages by the time required to identify the virus involved and then manufacture, test and distribute the resulting vaccine. Indeed, it is widely agreed that there is insufficient worldwide capacity to manufacture the amount of vaccine that will be required in a pandemic. Consequently, preparations for an effective public health response to deal with the onset of a pandemic in the absence of a vaccine are of crucial importance. Key elements of such a plan include the adequate provision of antiviral drugs and the implementation of effective non-pharmacological interventions, including infection control. However, within the framework of many national pandemic preparedness plans worldwide, there are considerable problems with the potential effectiveness of each of these elements.

Antiviral drugs will probably be the first line of defence in the event of an influenza pandemic and will be essential to contain the initial outbreak and reduce the extent of transmission and the severity of illness. Accordingly, both the WHO and many national governments are stockpiling the antiviral neuraminidase inhibitors oseltamivir and zanamivir as a core component of their pandemic response. In light of the importance of this form of intervention, and recent observations of oseltamivir resistance in $\mathrm{H} 5 \mathrm{~N} 1$ viruses, the decision taken by the UK government to only stockpile oseltamivir and not place an order for a second antiviral such as zanamivir seems foolhardy. Indeed, a recent report published by the Royal Society and the Academy of Medical Sciences (available at http://www.royalsoc.ac.uk) is highly critical of this position and urges the UK Department of Health to urgently "revisit the decision not to stockpile zanamivir at the same level" as oseltamivir. In addition, the report questions the decision to stockpile sufficient oseltamivir for only $25 \%$ of the UK population and asks whether this will be enough to treat all cases in a pandemic, even assuming the virus retains sensitivity to this drug.

A more fundamental concern is the paucity of options in the anti-influenza armamentarium. The only other class of antiviral agent currently licensed for the treatment of all strains of influenza is the amantadine-related compounds (M2 proton channel inhibitors), which cause neurological side-effects and readily select for resistant viruses. Consequently, the continued development of new anti-influenza dugs is of paramount importance. In this regard, the recent reports on peramivir, a new, more potent neuraminidase inhibitor that is scheduled to enter Phase II trials, are most welcome.

Before a vaccine becomes available, infection control and non-pharmacological interventions, including personal protective equipment and social distancing measures, will constitute a major element of pandemic control. Indeed, for many countries, these forms of intervention will be the only control measures available. Therefore, it is also worrisome that recent attempts to identify the most effective non-pharmacological strategies have revealed the lack of an appropriate level of supporting science. Undoubtedly, there has been increasing interest in these activities, including the recent award of grants by the CDC to address these issues in community settings. However, as this programme represents only $0.07 \%$ of their proposed pandemic influenza budget, significantly more financial commitment to optimize these types of intervention is urgently required, both in the United States and elsewhere.

Any plan that strives to arrive at a successful response to a challenge must be an evolving process that is informed by the best advice and knowledge available. A pandemic preparedness plan, where effective implementation of the right measures will be crucial to curtailing the devastating impact of a global disease outbreak, should be no different. 